\title{
Einfluss der Siedlungsstruktur auf das thermische Nutzungspotential von oberflächennahen Aquiferen
}

Zusammenfassung: Bereits bei der Bereitstellung der Heizwärme eines EinfamilienNiedrigenergiehauses mittels thermischer Nutzung des Aquifers wird das Grundwasser erheblich über die Grundstücksgrenzen hinaus thermisch beeinflusst (Temperaturanomalie). Dies hat zur Folge, dass in angrenzenden Gebieten eine thermische Nutzung des Grundwassers nur mehr eingeschränkt oder überhaupt nicht mehr möglich ist. Wie in dieser Arbeit gezeigt wird stellen vor allem in dicht besiedelten Gebieten die Ausdehnungen der Temperaturanomalien einen stark limitierenden Faktor für das nutzbare geothermische Potential dar. So können z. B. in einer dicht bebauten Einfamilienhaussiedlung mit guter Wärmedämmung nur rund $10 \%$ des Heizwärmebedarfs mithilfe einer thermischen Nutzung des Grundwassers abgedeckt werden.

The impact of housing patterns on the utilisation potential of the thermal energy of near-surface aquifers

Summary: Even where the thermal energy of an aquifer is used to heat only a singlefamily low-energy home, the temperature of the aquifer is substantially affected beyond its property boundaries (temperature anomaly). This implies that thermal usage in neighbouring areas becomes limited or even impossible. This study shows that such temperature anomalies tend to limit substantially the utilisable geothermal potential, especially in densely populated areas. In a closely packed housing development with well heat-insulated single-family homes, for example, only $10 \%$ of the heating requirements can be met by utilising the thermal energy of the groundwater.

\section{Einleitung}

Für Raumheizung, Klimaanlagen und Warmwasser werden in Österreich ungefähr $28 \%$ des gesamten energetischen Endverbrauchs aufgewendet. Die Errichtung von Gebäuden mit Niedrigenergiehaus und Passivhausstandard und die thermische Sanierung von Gebäuden zur Reduzierung des Energiebedarfs für Heizen und Kühlen sowie die Nutzung erneuerbarer Energien sind Schlüsseltechnologien zur Reduktion der Treibhausgasemissionen und damit wichtige Beiträge zum Klimaschutz.

Neben der Solarthermie ist die thermische Nutzung des Grundwassers/Untergrundes eine Schlüsseltechnologie die die Energie aus Sonne sowie aus dem Inneren der Erde nutzbar macht.

Bei der thermischen Nutzung des Untergrundes wird je nach Betrieb der Anlage dem Untergrund Wärme entzogen oder zugeführt.

Während sich bei der Nutzung des Untergrundes mit Hilfe von Erdwärmesonden das Erdreich überwiegend in vertikaler Richtung thermisch verändert, wird bei der thermischen Nutzung des Grundwassers die Temperatur überwiegend in horizontaler Richtung beeinflusst. Dies führt dazu, dass bereits für die Bereitstellung der Heizwärme eines Niedrigenergiehauses einer Familie mit durchschnittlichem Bedarf die thermische Änderung des Grundwassers, je nach Aquifereigenschaften erheblich über die Grundstücksgrenzen reicht. Somit ist eine thermische Nutzung des Grundwassers in angrenzenden Gebieten nur mehr eingeschränkt oder nicht mehr möglich und das theoretisch nutzbare Potenzial kann nicht vollständig ausgeschöpft werden.

In der Ermittlung des thermischen Nutzungspotentials der oberflächennahen
Aquifere bleibt die Siedlungsstruktur meist unberücksichtigt. Diese kann allerdings neben der tolerierbaren Beeinflussung des Grundwassers (d.h. die Temperaturveränderung ist vernachlässigbar gering) das Nutzungspotential beschränken. Für die Bewertung ist allerdings eine genaue Kenntnis über die thermische Veränderung (Temperaturanomalie) des Grundwassers und Untergrundes infolge der Rückgabe von thermisch genutztem Grundwassers erforderlich. Zur Abschätzung für Kleinanlagen existieren vereinfachte analytische Lösungen, z.B. die im ÖWAV-Regelblatt 207 (2009) verwendete analytische Berechnung der Ausbreitung Temperaturanomalien im Grundwasserstrom mittels einer iterativen Berechnung nach Ingerle (1988) unter der Annahme stationärer Bedingungen.

In Sitzenfrei et al. (2010) wird eine Datenbank für die Ausbreitung der Temperaturanomalien bei Kleinanlagen basierend auf einer detaillierten 3-dimensionalen numerischen Berechnung vorgestellt. Die Auswertung der Daten zeigte, dass die berechneten Ausbreitungen der Temperaturanomalien von den Ergebnissen einer Berechnung nach Ingerle erheblich abweichen können. Für diese Arbeit wurde diese Datenbank erweitert und dient als Basis für die Potentialanalyse.

Für das (regionale) thermische Nutzungspotential von oberflächennahen Aquiferen wird ein umfassender Überblick in Rauch und Stegner (2004) gegeben. Darin wurde aus Literaturhinweisen abgeleitet, dass bei einer Veränderung

TABELLE 1

Bebauung

(GSF= Grundstückfläche GRF= Grundfläche und BGF=Bruttogeschossfläche)

\begin{tabular}{lccccc} 
Bebauung & GSF & GRF & BGF & Dichte GRF & Dichte BGF \\
\hline Einfamilienhaus (EF1) & 800 & 160 & 160 & $10 \%$ & $10 \%$ \\
\hline Einfamilienhaus (EF2) & 400 & 160 & 160 & $27 \%$ & $27 \%$ \\
\hline Mehrfamilienhaus (MF) & 400 & 120 & 240 & $20 \%$ & $40 \%$ \\
\hline Zeilenbebauung 3 gesch. (Z3) & 720 & 240 & 720 & $16 \%$ & $49 \%$ \\
\hline Zeilenbebauung 5 gesch. (Z5) & 1092 & 240 & 1200 & $12 \%$ & $63 \%$ \\
\hline gemischt 1 (G1) & - & - & - & $18 \%$ & $47 \%$ \\
\hline gemischt 2 (G2) & - & - & - & $8 \%$ & $22 \%$ \\
\hline
\end{tabular}


der Grundwassertemperatur um $3 \mathrm{~K}$ keine signifikanten Auswirkungen in ökologischer, chemischer und mikrobieller Hinsicht zu erwarten sind. Für die regionale Veränderung des Grundwassers gibt es in Österreich keine konkreten Vorgaben, im Schweizer Wasserrecht wird die Veränderung mit $3 \mathrm{~K}$ beschränkt (GSCHV 2008). Diese Beschränkung wird auch in der folgenden Arbeit für die Berechnung des Nutzungspotentials herangezogen. Des weiteren wird in Rauch und Stegner (2004) auch eine Methodik zur Bewertung des thermischen Potentials großräumiger Aquifere basierend auf einer Grundwasserwärmebilanzierung vorgestellt. Im Folgenden wird diese für die Berechnung der mittleren Temperaturabsenkung im betrachteten Aquifer herangezogen.

Ziel dieser Arbeit ist die Erhebung des möglichen Versorgungsgrades sowie des Potentials der Grundwasserwärmenutzung zur Bereitstellung von Raumwärme unter Berücksichtigung der Siedlungsstruktur und der lokalen Temperaturanomalien.

\section{Methodik}

\subsection{Siedlungsstruktur}

Für die Beurteilung des Einflusses der Siedlungsstruktur auf das nutzbare thermische Potential werden $1000 \mathrm{~m}$ auf $1000 \mathrm{~m}$ große (virtuelle) Siedlungsgebiete mit unterschiedlichen Siedlungsstrukturen untersucht. Auf fünf Siedlungsgebieten werden die in Reinhardt und Trudel (1979) vorgeschlagenen Bebauungsformen monoton platziert (Tab. 1). Für zwei weitere Siedlungsgebiete werden Ausschnitte aus einer realen Bebauung ausgewählt (Abb. 1).

Für die (virtuellen) Siedlungsgebiete wird das Untersuchungsgebiet in $\sim 120 \mathrm{~m}$ auf $120 \mathrm{~m}$ große Baublöcke unterteilt. Die Baublöcke werden anschließend in Grundstücke aufgeteilt und das Gebäude wird mittig am Grundstück platziert. Für die gemischte Siedlungsstruktur werden die Grundrisse aus einer bestehenden Siedlung verwendet. Hierzu wurden zwei $1000 \mathrm{~m}$ auf $1000 \mathrm{~m}$ große Teilgebiete mit unterschiedlicher Besiedelungsdichte ausgewählt. Die Bruttogeschossfläche (BGF) wurde aufgrund der Grundfläche (GRF) der Gebäude abgeschätzt, für Gebäude $<160 \mathrm{~m}^{2}$ GRF ergibt sich die BGF zu 2 x GAF für Gebäude mit einer GRF $>160 \mathrm{~m}^{2}$ zu $3 \mathrm{x}$ GAF.

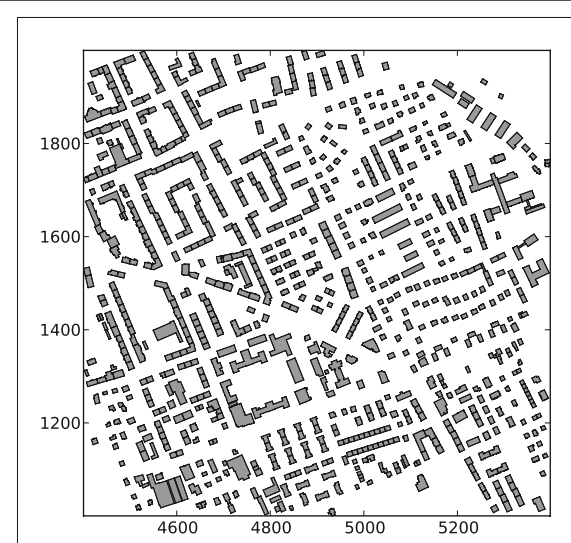

[m]

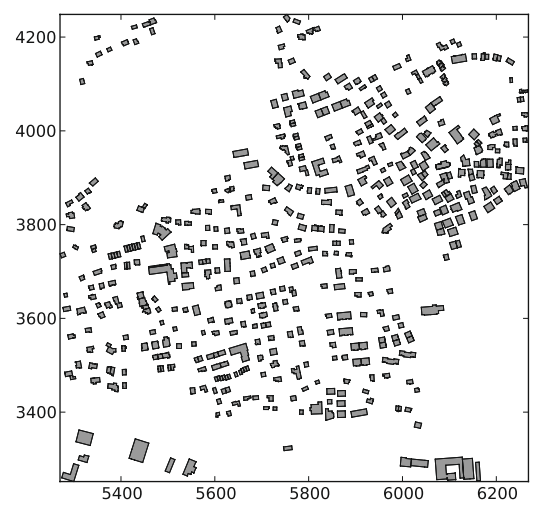

[m]

Abb. 1: Siedlungsstruktur: links G1, rechts G2.

\begin{tabular}{|c|c|c|c|c|c|c|c|c|}
\hline$=======$ & $\mathrm{kf}(\mathrm{m} / \mathrm{s})$ & $\operatorname{kfHzuV}(-)$ & $Q\left(\mathrm{~m}^{3} / \mathrm{s}\right)$ & $\begin{array}{r}\text { TempR( }\left({ }^{\circ} \mathrm{C}\right) \\
=======\end{array}$ & ) $B D(h)$ & $\operatorname{Disp}(\mathrm{m})$ & $L(m)$ & $\begin{array}{l}B(\mathrm{~m}) \\
=====\end{array}$ \\
\hline $\begin{array}{l}0.000250 \\
0.000250 \\
0.000250 \\
0.000250 \\
0.000250 \\
0.000250 \\
0.001000\end{array}$ & $\begin{array}{l}0.05000 \\
0.05000 \\
0.00500 \\
0.00500 \\
0.00050 \\
0.00050 \\
0.05000\end{array}$ & $\begin{array}{cc}00 & 10 \\
00 & 2 \\
00 & 10 \\
00 & 2 \\
00 & 10 \\
00 & 2 \\
00 & 10\end{array}$ & $\begin{array}{l}0.000500 \\
0.000500 \\
0.000500 \\
0.000500 \\
0.000500 \\
0.000500 \\
0.000500\end{array}$ & $\begin{array}{l}5 \\
5 \\
5 \\
5 \\
5 \\
5 \\
5\end{array}$ & $\begin{array}{l}2000 \\
2000 \\
2000 \\
2000 \\
2000 \\
2000 \\
2000\end{array}$ & $\begin{array}{l}1 \\
1 \\
1 \\
1 \\
1 \\
1 \\
1\end{array}$ & $\begin{array}{l}144.15 \\
83.69 \\
96.46 \\
72.44 \\
45.96 \\
43.35 \\
158.38\end{array}$ & $\begin{array}{r}15.99 \\
9.26 \\
102.00 \\
31.22 \\
60.16 \\
51.27 \\
7.02\end{array}$ \\
\hline
\end{tabular}

Abb. 2: ASCII Datenbank

Für jedes Siedlungsgebiet werden je drei Situationen untersucht wobei die Gebäude jeweils einem der nachfolgenden Standards entsprechen (AB 207):

- Altbau mit unzureichendere Wärmedämmung nicht dem Stand der Technik entsprechend (ca. $75 \mathrm{~W} / \mathrm{m}^{2}$ )

- Neubau mit guter Wärmedämmung (ca. $40 \mathrm{~W} / \mathrm{m}^{2}$ )

- Passivhaus (ca. 15W/m²)

Im nächsten Schritt wird der Wärmebedarf für die Heizleistung für jedes Gebäude ermittelt. Die erforderliche Heizleistung wurde aus den spezifischen Heizleistungen (s. o.) und unter der Annahme von 2000 Volllaststunden pro Jahr berechnet. Anschließend wird die erforderliche Wassermenge berechnet (Spreizung $\Delta \mathrm{T}=-3 \mathrm{~K})$.

Für die Platzierung der Anlagen wird ein Wärmelastplan erstellt in dem für jede Anlage der Entnahme- und Rückgabebrunnen sowie die zugehörigen Temperaturfahnen und hydraulisch beeinflussten Bereiche eingetragen werden. Um einen nachhaltigen und konfliktfreien Betrieb der Einzelanlagen zu gewährleisten, werden keine Überschei- dungen von Temperaturfahnen und hydraulisch beeinflussten Bereichen zugelassen. Kann eine Anlage nicht konfliktfrei platziert werden, wird sie nicht installiert.

Zusätzlich zu den Einzelanlagen für jedes Gebäude wird untersucht ob durch eine gemeinsame Nutzung einer Geothermieanlage durch alle Gebäude innerhalb eines Baublocks das nutzbare geothermische Potential gesteigert werden kann.

\subsection{Berechnung der Temperatur- anomalien}

Die Berechnung der Ausdehnung der Temperaturfahne hat einen entscheidenden Einfluss auf die Ergebnisse. Da eine detaillierte physikalische Berechnung für eine Potentialerhebung aufgrund der aufwendigen Berechnungsmodelle nicht oder zumindest nur schwer möglich ist wird auf ein vereinfachtes Verfahren zurückgegriffen.

Die Beeinflussung des Grundwassers aufgrund der thermischen Nutzung reicht theoretisch bis ins Unendliche, weshalb für die Ausbreitung der Temperaturano- 

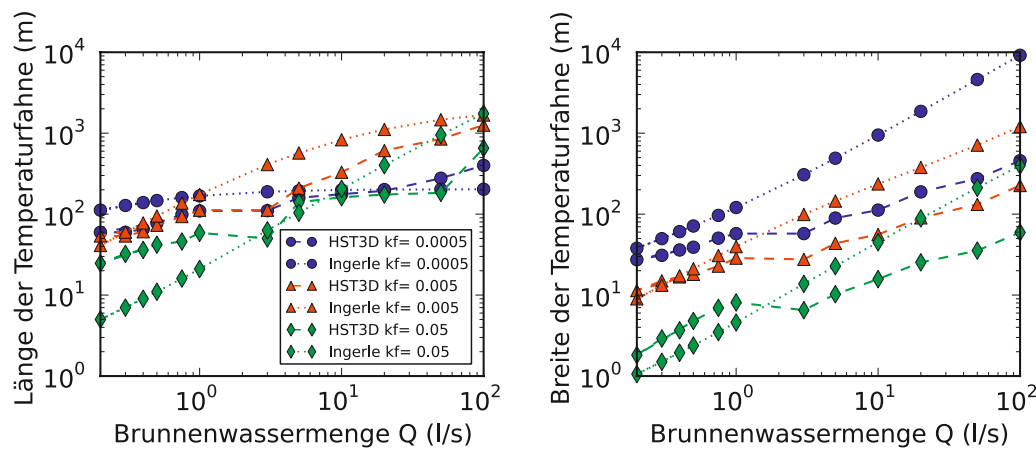

Abb. 3: Vergleich der Temperaturfahnen: I = 0,001 m/m; Betriebsdauer $2000 \mathrm{~h}$.

TABELLE 2

Parameterbereiche für Aquifereigenschaften

\begin{tabular}{lcl} 
Parameter & Bereich & Einheit \\
\hline Flurabstand $(\mathrm{A})$ & 5 & $\mathrm{~m}$ \\
\hline Grundwassermächtigkeit $(\mathrm{H})$ & 25 & $\mathrm{~m}$ \\
\hline Grundwasserspiegelgefälle $(\mathrm{I})$ & 1 & $\mathrm{~mm} / \mathrm{m}$ \\
\hline Hydraulische Durchlässigkeit $\left(\mathrm{k}_{\mathrm{f}}\right)$ & 0,005 & $\mathrm{~m} / \mathrm{s}$ \\
\hline Horizontale zu vertikale Durchlässigkeit & 0,0005 & - \\
\hline Longitudinale Dispersion & 2 & $\mathrm{~m}$ \\
\hline Wärmeleitfähigkeit $\lambda \mathrm{D}$ & 10 & $\mathrm{~W} / \mathrm{m}^{2}$ \\
\hline Porenzahl & 1,0 & $(-)$ \\
\hline & 0,2 &
\end{tabular}

malie der technische Grenzwert ab dem das Grundwasser als unbeeinflusst gilt mit einem Temperaturunterschied von $\Delta \mathrm{T}=1 \mathrm{~K}$ angesetzt wird.

In Sitzenfrei et al. (2010) wurde eine Datenbank (siehe Abb. 2) für kleine Anlagen mit Brunnenwassermengen von 0,5$1,0(1 / \mathrm{s})$ vorgestellt, die einen großen Parameterraum üblicher Bodenkennwerte abdeckt. Insgesamt wurden darin 864 unterschiedliche Parameterkonfigurationen numerisch untersucht. Die berechneten Werte für die Längen und Breiten der Wärmefahnen beruhen auf den Ergebnissen des 3-dimensionalen finiten Differenzen Modell HST3D (Kipp, Jr. 1997) für gekoppelten Fluid-, Stoff- und Wärmetransport.

Ein Vergleich der Temperaturfahnen mit der vereinfachten analytischen Berechnung nach Ingerle (1988) zeigt teils erhebliche Abweichungen vor allem bei der Breite der Temperaturfahnen (siehe Abb. 3).

Die Berechnung der Ausdehnung der Temperaturfahnen wird mit Hilfe der Datenbank, die für diesen Zweck auf einen Wertebereich von $0,2(\mathrm{l} / \mathrm{s})$ für Kleinanlagen und bis zu $100(1 / \mathrm{s})$ für Großanlagen erweitert wurde, durchgeführt.

\subsection{Hydraulische Berechnung}

Für die Berechnung des erforderlichen horizontalen Abstandes zwischen Entnahmebrunnen und Rückgabebrunnen (zur Vermeidung von thermischen Kurzschlüssen) und des hydraulisch beeinflussten Bereiches wird auf die empirischen Formel von Möderl et al. (2010) zurückgegrifauf der Auswertung der Ergebnisse des 3-dimensionalen finiten Differenzen Modells HST3D.

\subsection{Berechnung der regionalen Beanspruchung}

Zur Beurteilung der thermischen Belastung wird mithilfe der in Rauch und Stegner (2004) vorgestellten Methodik eine vereinfachte Wärmebilanz über das Siedlungsgebiet gebildet und die durchschnittliche Temperaturänderung berechnet. Als Grenzwert für die regional zulässige Temperaturveränderung des Aquifers wird die Vorgabe aus dem Schweizer Wasserrechts GSCHV, 28. Oktober 1998 (Stand am 1. Juli 2008) mit maximal $3 \mathrm{~K}$ übernommen. Bis zu diefen. Die Abstandsformel basiert ebenfalls sem Grenzwert sind keine signifikanten negativen Auswirkungen in ökologischer, chemischer und mikrobieller Sicht zu erwarten.

Die Grundwasserwärmebilanz wird anhand eines Kontrollvolumens erstellt, die Transportvorgänge werden wie folgt unterteilt:

- Konvektiver Energietransport $P_{K}$

- Wärmeleitung $P_{L}$

- Dispersiver Transport $P_{D}$

- Wärmespeicherung $\mathrm{P}_{\mathrm{W}}$

\subsubsection{Konvektiver Energietransport $P_{K}$}

$\mathrm{Zu}$ einem konvektiven Wärmetransport kommt es bei allen Wasser $\mathrm{Zu}$ - und $\mathrm{Ab}$ flüssen in das Kontrollvolumen. Der Energieeintrag berechnet sich wie folgt:

$$
P_{K}=c_{V W} \cdot Q \cdot T_{E}
$$

$c_{V W} \ldots$ spezifische Wärmekapazität $\left(\mathrm{J} / \mathrm{m}^{3} \mathrm{~K}\right)$

$T_{E} \ldots$ Temperatur in (K)

$Q \ldots$ Grundwasserstrom $\left(\mathrm{m}^{3} / \mathrm{s}\right)$

Für das hier verwendete Kontrollvolumen wird nur der $\mathrm{Zu}$ - und Abfluss von Grundwasser berücksichtigt.

\subsubsection{Wärmeleitung $P_{L}$}

Zur Abschätzung des Wärmeeintrages durch Wärmeleitung in das Kontrollvolumen wird auf die vereinfachte Berechnung nach Rauch und Stegner (2004) zurückgegriffen. Der Energiestrom zufolge von Wärmeleitung ergibt sich zu:

$P_{L}=\frac{\lambda_{D} \cdot F \cdot \Delta T}{A+\frac{H}{4}}$

mit

$\lambda_{D} \quad$ Wärmeleitfähigkeit des Bodens $\left(\mathrm{W} / \mathrm{m}^{2} \mathrm{~K}\right)$

$\Delta T \quad$ Temperaturänderung (K)

A Flurabstand (m)

$H \quad$ Grundwassermächtigkeit (m)

$F \quad$ horizontale Fläche des betrachteten Gebietes $\left(\mathrm{m}^{2}\right)$

Bei der Rückgabe von kleinen Wassermengen in einen mächtigen Grundwasserkörper erfolgt die Rückgabe meist nicht homogen über die gesamte Höhe. Wie beim Berechnungsverfahren nach Ingerle ist als maßgebliche Grundwassermächtigkeit zumindest die Distanz von Grundwasserspiegel bis zur Unterkante des Brunnens anzusetzen. (siehe ÖWAVRegelblatt 207, 2009) 


\subsubsection{Dispersiver Wärmetransport $P_{D}$}

Der dispersive Wärmetransport wird in der Bilanz nicht berücksichtig, da die Temperaturfahnen nicht über die Grenzflächen des Kontrollvolumens reichen und der Wärmetransport über Dispersion für den verbleibenden Grundwasserkörper keine relevante Größe darstellt.

\subsubsection{Wärmespeicherung $P_{w}$}

Die geothermische Nutzung des Grundwassers führt zu einer Verringerung oder Erhöhung der gespeicherten Wärmeenergie.

$$
P_{W}=c_{V W} \cdot V \cdot \frac{\Delta T}{\Delta t}
$$

$c_{V W} \quad$ spezifische Wärmekapazität $\left(\mathrm{J} / \mathrm{m}^{3} \mathrm{~K}\right)$

$V \quad$ Volumen $\left(\mathrm{m}^{3}\right)$

$\Delta T / \Delta t$ Temperaturveränderung im Kontrollvolumen je Zeiteinheit

Die Wärmebilanzgleichung ergibt sich somit aus den Energieströmen zu:

$$
P_{W}=\sum P_{K}+P_{L}+P_{D}
$$

Die regionale Beanspruchung der Aquifere - mittlere Temperaturveränderung lässt sich durch Lösen dieser Wärmebilanzgleichung unter Vernachlässigung der Dispersion wie folgt berechnen:

$$
\begin{aligned}
& c_{V W} \cdot V \cdot \frac{T}{\Delta t}=\frac{\lambda_{D} \cdot F \cdot \Delta T}{A+\frac{H}{4}} \\
& +c_{V W} \cdot Q \cdot \Delta T
\end{aligned}
$$

\subsection{Simulationen}

Es werden für jede Bebauung und für jeden Gebäudestandard je 100 Simulationen mit den in Tabelle 2 angeführten Parametern durchgeführt.

Des Weiteren wird untersucht, ob sich der Versorgungsgrad durch eine gemeinschaftliche Nutzung von Geothermieanlagen verändert. Dafür werden für jede Bebauung mit Monostruktur (Tab. 1: EF1, EF2, MF, Z3, Z5) für jeden Gebäudestandard je 1000 Simulationen durchgeführt. Für jede Simulation wird zufällig ein Gruppierungsfaktor zwischen 0 und $100 \%$ ermittelt, der angibt wie viele Baublöcke eine gemeinschaftliche Geothermieanlage nutzen (d. h. bei $100 \%$ werden alle Baublöcke durch eine Anlage versorgt).

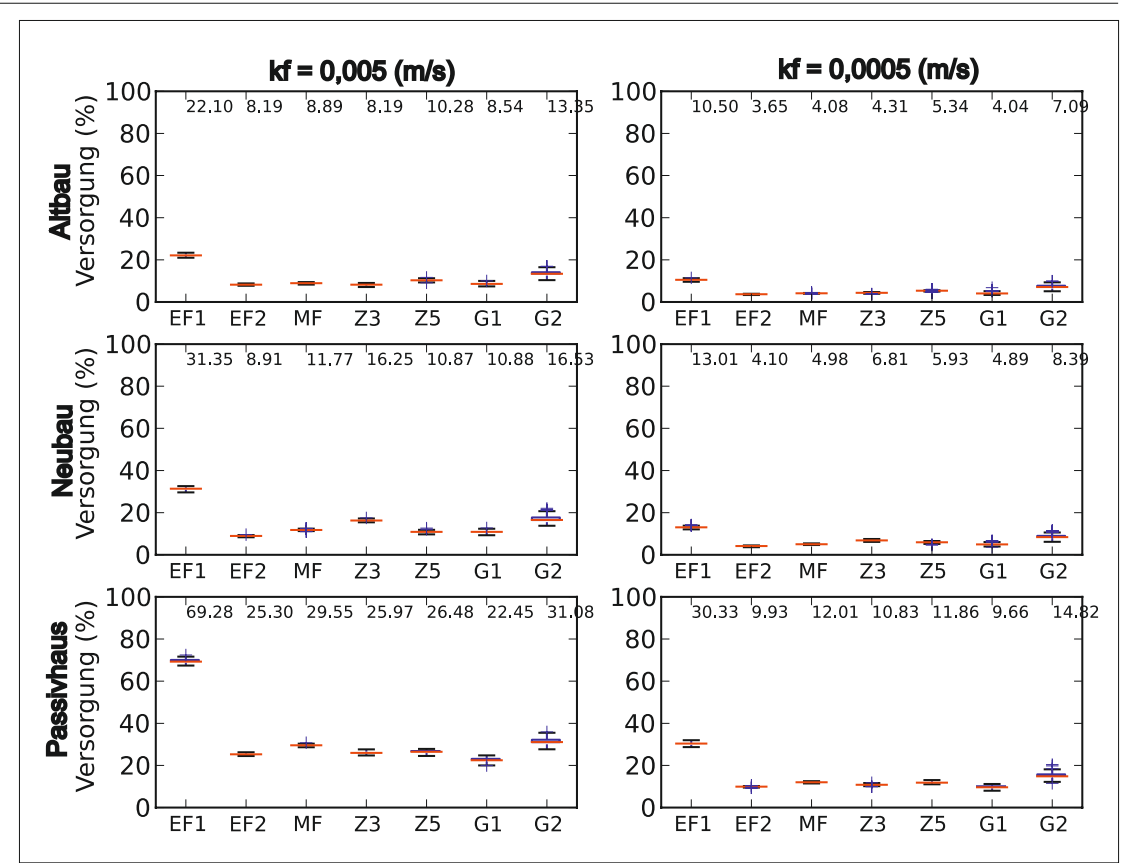

Abb. 4: Versorgungsgrade.

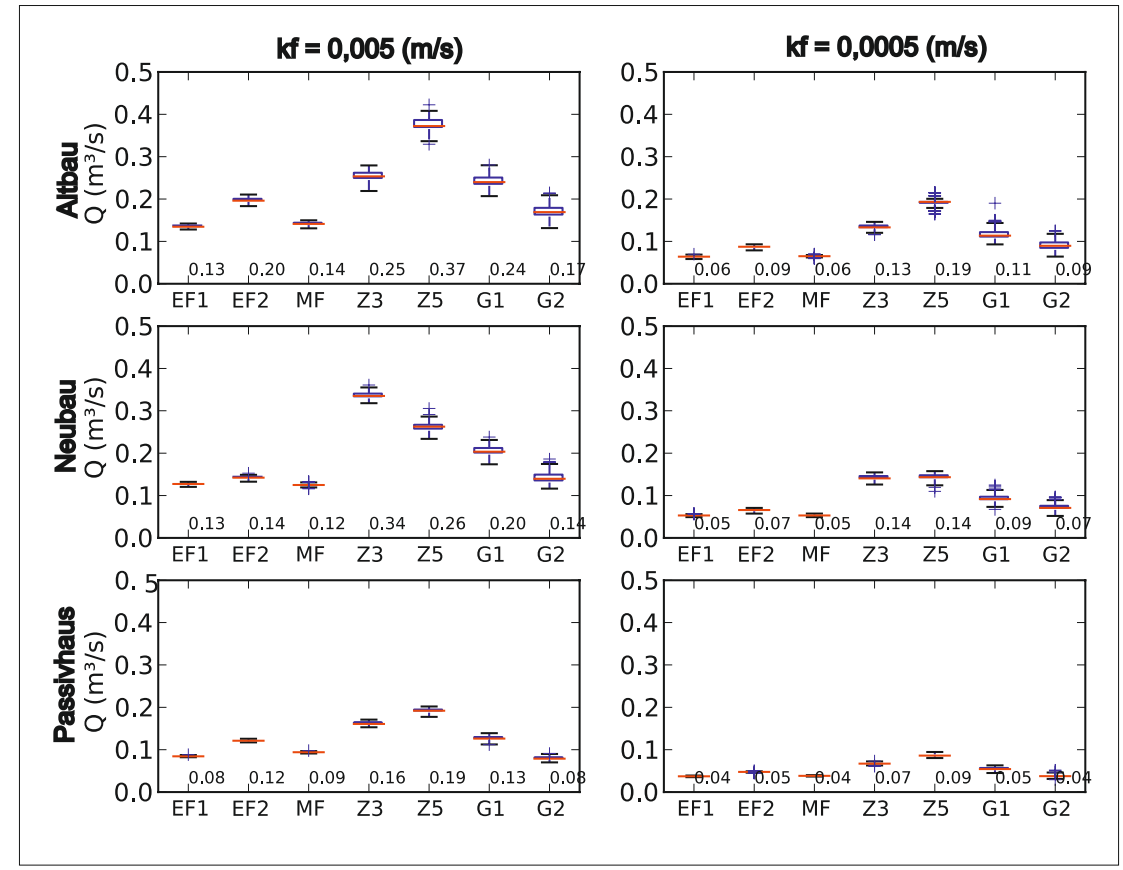

Abb. 5: thermisch genutzte Wassermenge.

\section{Resultate}

In Abbildung 4 ist der Versorgungsgrad für die Bebauungen für unterschiedliche Gebäudestandards sowie kf-Werte unter der Annahme, dass jedes Gebäude versucht eine eigene Geothermieanlage zu betreiben, als Boxplot dargestellt. Des Weiteren werden die Medianwerte für die erreichten Versorgungsgrade in (\%) angegeben. Der Versorgungsgrad gibt den Prozentsatz des Heizbedarfs an, der mittels thermi- scher Nutzung gedeckt werden kann. Die jeweiligen Boxen in der Abbildung repräsentieren die Werte innerhalb der $25 \%$ und $75 \%$ Quartil, die rote Markierung stellen den Medianwert dar, die Whisker (schwarzen Balken) reichen bis zum 1,5-fachen des Interquartilsabstands und die blauen Markierungen außerhalb der Boxen zeigen die Ausreißer an.

Aus den Medianwerten wird ersichtlich, dass eine Reduktion des Bedarfes nicht direkt in eine Erhöhung des Versor- 


\begin{tabular}{|c|c|c|c|c|}
\hline Bebauung & $\begin{array}{l}\text { Dichte GRF } \\
\text { (\%) }\end{array}$ & $\begin{array}{l}\text { Bedarf } \\
\text { (MW) }\end{array}$ & $\begin{array}{l}\text { Bedarfs- } \\
\text { deckung (\%) }\end{array}$ & $\begin{array}{l}\text { genutzte } \\
\text { Wassermenge } \\
\left(\mathrm{m}^{3} / \mathrm{s}\right)\end{array}$ \\
\hline EF1 & 10 & 4,0 & 31,35 & 0,13 \\
\hline EF2 & 27 & 10,8 & 8,91 & 0,14 \\
\hline MF & 20 & 16,0 & 11,77 & 0,12 \\
\hline Z3 & 16 & 19,6 & 16,25 & 0,34 \\
\hline Z5 & 12 & 25,2 & 10,87 & 0,26 \\
\hline G1 & 18 & 18,8 & 10,88 & 0,20 \\
\hline G2 & 8 & 8,8 & 16,53 & 0,14 \\
\hline
\end{tabular}

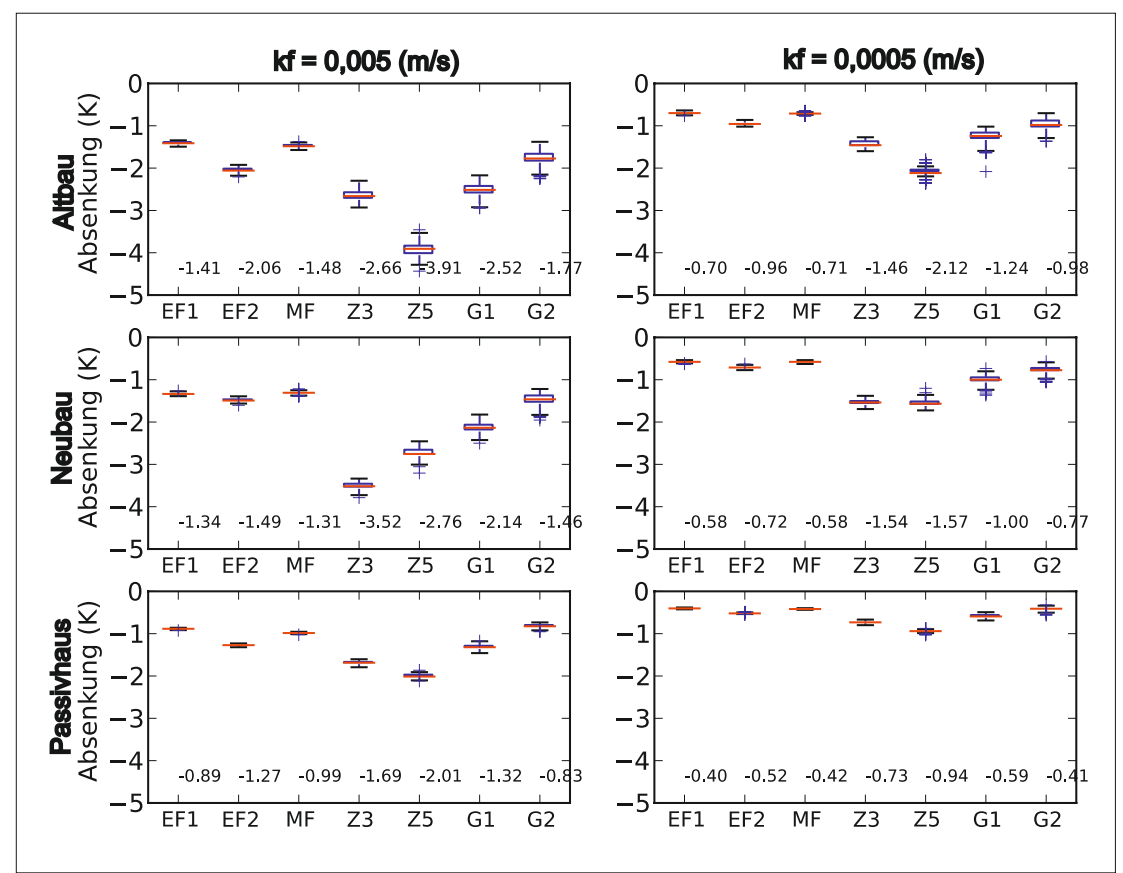

Abb. 6: Mittlere Temperaturveränderung des Aquifers.

gungsgrades umgerechnet werden kann. Der Wärmebedarf wurde z. B. für den Gebäudestandard Altbau mit $75 \mathrm{~W} / \mathrm{m}^{2}$ angesetzt und für das Passivhaus mit $15 \mathrm{~W} / \mathrm{m}^{2}$. Unter der Annahme gleichbleibender geothermischer Ressourcen würde sich daraus theoretisch eine Verfünffachung des Versorgungsgrades ergeben. Allerdings ergibt sich für die Bebauung EF2 mit dem Gebäudestandard Altbau laut Medianwert ein Versorgungsgrad $8,19 \%$ und für den Passivhausstandard von 25,30\% und somit lediglich eine Steigerung um das Dreifache. Des Weiteren wird ersichtlich, dass der Versorgungsgrad stark vom kf-Wert abhängig ist. So kann bei einem kf-Wert von 0,0005 (m/s) nur rund die Hälfte des Versorgungsgrades der sich für einen kf-Wert von $0,005(\mathrm{~m} / \mathrm{s})$ ergibt erreicht werden.
$\mathrm{Q}_{\mathrm{m}} \mathrm{zu} 0,038 \mathrm{~m}^{3} / \mathrm{sK}$. Unter der Annahme der den Simulationen zugrundeliegenden 2000 Volllaststunden ergibt sich $\mathrm{Q}=0,16 \mathrm{~m}^{3} / \mathrm{sK}$. Somit ergibt sich bei einer regionalen mittleren Absenkung der Grundwassertemperatur um $3 \mathrm{~K}$ die nutzbare Wassermenge $\mathrm{zu}$ $0,48 \mathrm{~m}^{3} / \mathrm{s}$.

Die nutzbare theoretische Wassermenge wird von keiner der ausgewerteten Simulationen in Abbildung 5 erreicht. Aus Abbildung 4 und Abbildung 5 wird ersichtlich, dass nicht das nutzbare geothermische Potential den limitierenden Faktor für den Versorgungsgrad darstellt sondern die Ausdehnung der Temperaturfahnen.

In Tabelle 3 sind die Medianwerte aus Abbildung 4 und Abbildung 5 für den Gebäudestandard Neubau und den kf-Wert von $0,005 \mathrm{~m} / \mathrm{s}$ den GRF Dichten und dem Bedarf gegenübergestellt. Es wird ersichtlich, dass sich für die höchste Bebauungsdichte (EF2) der geringste Versorgungsgrad ergibt obwohl der Bedarf an Heizwärme z.B. für die fünf geschoßige zeilenförmige Bebauungen doppelt so hoch ist. Man erkennt, dass unabhängig vom gesamten Bedarf rund 10-16\% des Bedarfs mittels einer thermalen Nutzung des Grundwassers abgedeckt werden können lediglich für EF1 ergibt sich aufgrund der geringen Bebauungsdichte eine höhere Bedarfsdeckung.

In Abbildung 6 ist die mittleren Absenkungen der Grundwassertemperatur dargestellt. Die eingetragenen Werte stellen Medianwert dar. Wie aus der Abbildung ersichtlich ist wird in den meisten Fällen der Grenzwert im Median für die Beeinflussung der Grundwassertemperatur unterschritten. Lediglich für die Bebauung Z5 nach dem Gebäudestandard Altbau sowie Z3 Gebäudestandard Neubau für einen kf-Wert von 0,005 wird das $3 \mathrm{~K}$ Kriterium nicht erfüllt.

In Abbildung 7 sind die Wärmelastpläne für die Bebauung G1 und G2 mit dem Gebäudestandard Neubau dargestellt. Man sieht deutlich, dass bereits Kleinanlagen erhebliche Temperaturfahnen erzeugen und die Nutzung des Grundwassers zu Heizzwecken für benachbarte Gebäude (unter der Annahme dass sich die Temperaturfahnen nicht überscheiden dürfen) nicht mehr möglich ist. Die Ausdehnung der Temperaturfahnen wurde für einen ungestörten Zustand d. $h$. ohne Interaktion mit benachbarten Geothermieanlagen berechnet. Wie aus der Abbildung ersichtlich wird kann eine gegenseitige Beeinflussung bei starker Beanspruchung des Aquifers nicht ausgeschlos- 


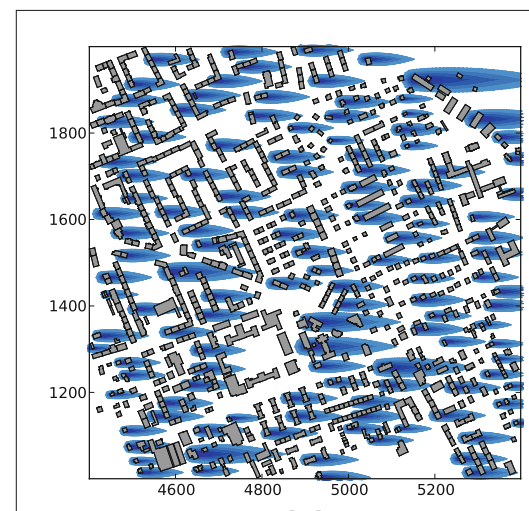

[m]

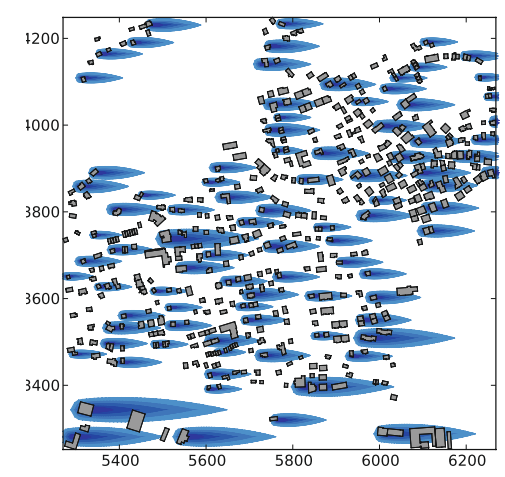

[m]
Abb. 7: Wärmelastpläne links für G1 und rechts für G2 für den Gebäudestandard Neubau.

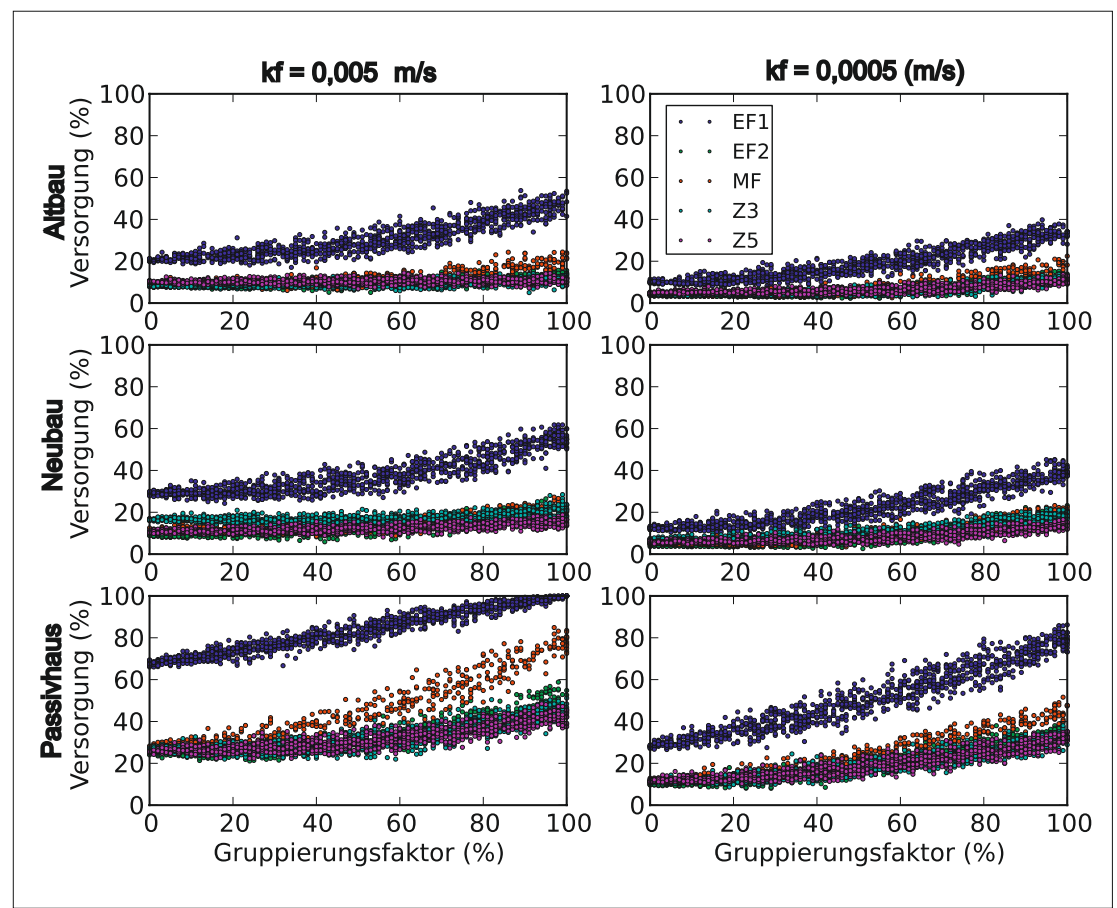

Abb. 8: Versorgungsgrad für gruppierte Anlagen.

sen werden. Dies sollte in zukünftigen Berechnungen berücksichtigt werden.

In Abbildung 8 sind die Simulationsergebnisse zur Untersuchung des Einsatzes gruppierter Anlagen - Gebäude innerhalb eines Baublocks nutzen eine große Geothermieanlage - für die Bebauungen EF1, EF2, MF, Z3 und Z5 für die unterschiedlichen Gebäudestandards und kfWerte eingetragen. Auf der Abszisse ist der Gruppierungsfaktor und auf der Ordinate der Versorgungsgrad dargestellt. Wie ersichtlich wird kann der Versorgungsgrad durch die Nutzung größerer Geothermieanlagen deutlich erhöht werden. Allerdings wird dann in den meisten Fällen die Temperatur über das 3 K Kriterium abgesenkt.

\section{Zusammenfassung}

Für die Untersuchung des Einflusses der Siedlungsstruktur auf das thermische Nutzungspotential eines oberflächennahen Aquifers wurden insgesamt sieben unterschiedliche Siedlungsgebiete mit den Abmessungen 1000 x 1000 m untersucht. Fünf Siedlungsgebiete wurden monoton mittels den in Reinhardt und Trudel (1979) vorgeschlagenen Bebauungsformen bebaut. Für die verbleibenden zwei Siedlungsgebiete wurden zwei Ausschnitte aus einer realen Bebauung ausgewählt.

Für die Siedlungen wurden je der Gebäudestandard Altbau, Neubau und Passivhaus untersucht. Anschließend wurde versucht für jedes Gebäude eine Geothermieanlage konfliktfrei zu platzieren. Konfliktfrei heißt in diesem Zusammenhang, dass keine Überschneidungen von hydraulisch oder thermisch beeinflussten Bereichen zugelassen werden. Um die Auswirkungen verschiedener Konfigurationen zu untersuchen wurden für jedes Untersuchungsgebiet 100 Simulationen mit einer zufälligen Reihenfolge der nacheinander zu plazierenden Geothermieanlagen erstellt, in Summe 7.000 Simulationen. Des Weiteren wurde der Einsatz gruppierter Anlagen - Gebäude innerhalb eines Baublocks nutzen gemeinsam eine große Geothermieanlage untersucht. Hierfür wurde für die fünf monoton bebauten Siedlungsgebiete der Anteil der gruppierten Anlagen zwischen $0 \%$ und $100 \%$ variiert. Es wurden je 1.000 Simulationen durchgeführt, somit ergeben sich in Summe 30.000 Simulationen.

Aus den durchgeführten Simulationen wurde ersichtlich, dass die Siedlungsstruktur einen erheblichen Einfluss auf das nutzbare Potential eines oberflächennahen Aquifers hat. Es wurde gezeigt, dass eine Verringerung des Heizwärmebedarfs nicht direkt in eine Erhöhung des Versorgungsgrades umgerechnet werden kann. So erhöht sich die Bedarfsdeckung bei einer Reduktion des Heizwärmebedarfs auf ein Fünftel z. B. von Altbau mit unzureichenderer Wärmedämmung nach Passivhaus lediglich um das Dreifache. Des Weiteren wurde gezeigt, dass der Versorgungsgrad sehr stark vom kf-Wert abhängig ist. Die Bedarfsdeckung ist für einen kf-Wert von $0,0005 \mathrm{~m} / \mathrm{s}$ nur halb so groß wie bei einem kf-Wert von $0,005 \mathrm{~m} / \mathrm{s}$.

Es wurde ersichtlich, dass sich das volle geothermische Potential nur ausschöpfen lässt wenn, die Geothermieanlagen in Größe und Lage mithilfe von Wärmelastplänen unter Berücksichtigung zukünftiger Anlagen günstig platziert werden.

Die Qualität der Wärmelastpläne ist sehr stark von der gewählten Berechnungsmethode der Temperaturfahnen abhängig. Wie in Sitzenfrei et al. (2010) gezeigt wurde, stimmen die berechneten Ergebnisse nach den unterschiedlichen Berechnungsverfahren nur bedingt überein. Des Weiteren bleiben in den herkömmlichen Berechnungsverfahren die sehr komplexen Interaktionen zwischen benachbarten Geothermieanlagen unberücksichtigt, die einen erheblich Einfluss für die geothermische Nutzung haben können. Hierfür fehlen noch geeignete Berechnungsverfahren die sich auf regionaler Ebene anwenden lassen. Im Rah- 
men des Forschungsprojektes GEOPOT werden diese effizienten Berechnungsverfahren für die thermische Nutzung des Untergrundes und Grundwassers entwickelt um einen nachhaltigen und konfliktfreien Betrieb der Geothermieanlagen in einem stark beanspruchten Aquifer zu gewährleisten.

\section{Danksagung}

Die Autoren bedanken sich bei der Österreichischen Forschungsförderungsgesellschaft mbH sowie beim Klima und Energiefond, für die Förderung dieser Arbeit im Rahmen des Forschungsprojektes GEOPOT P818890 und des Forschungs- projektes PowerVIBe P818878. (Dieses Projekt wird aus Mitteln des Klima- und Energiefonds gefördert und im Rahmen des Programms „NEUE ENERGIEN 2020“ durchgeführt.)

\section{Korrespondenz:}

Arbeitsbereich Umwelttechnik - Universität Innsbruck

Technikerstrasse 13

6020 Innsbruck

Tel.: (0043-512) 5076956

E-Mail: christian.urich@uibk.ac.at

DI Robert Sitzenfrei

DI Dr. Michael Möderl

Univ. Prof. DI. Dr. Wolfgang Rauch

Arbeitsbereich Umwelttechnik - Universität Innsbruck

Technikerstrasse 13

6020 Innsbruck

\section{LITERATUR}

GSCHV (28. Oktober 1998 [Stand am 1. Juli 2008]) Gewässerschutzverordung

Ingerle K (1988) Beitrag zur Berechnung der Abkühlung des Grundwasserkörpers durch Wärmepumpen. In: Österreichische Wasserwirtschaft, Vol. Jg. 40 H. 11/12

Kipp, Jr KL (1997) Guide to the Revised Heat and Solute Transport Simulator HST3D - Version 2. In: Water Resources Investigations Report 974157. U.S. Geological Survey.

Möderl M, Sitzenfrei R, Rauch W (2010) Empirical equation for spacing of groundwater heat pump systems; In: Proceedings of the World Environmental \& Water Resources Congress 2010: Challenges of Change, Providence, Rhode Island, 16-20 Mai 2010

ÖWAV-Regelblatt 207 (2009) ÖWAV-Regelblat
207 Thermische Nutzung des Grundwassers und des Untergrundes - Heizen und Kühlen Rauch W, Stegner U (2004) Das thermische Nutzungspotential von oberflächennahen Aquiferen aus wasserwirtschaftlicher Sicht. In: GWF - Wasser/Abwasser, Vol. 145 (2004)(5), S. 318Reinhardt W, Trudel H (1979) Wohndichte und Bebauungsformen Praktische Entscheidungshilfen für die kommunale Planung. Veröffentlichung der Forschungsgemeinschaft Bauen und Wohnen Nr. 113, Stuttgart

Sitzenfrei R, Möderl M, Urich C, Rauch W (2010) Thermal regeneration of aquifers for sustainable resource management; In: Proceedings of the Austria national young water professionals conference, Vienna, Austria, 9-11 Juni 2010 